\title{
Efecto de la cirugía de catarata en la sensibilidad al contraste y la calidad de vida de pacientes con diferentes tipos de catarata
}

\section{Effect of cataract surgery on contrast sensitivity and quality of life in patients with different types of cataract}

\author{
Valentina Berríos-Dolz ${ }^{1}$, Patricia Chirinos-Saldaña ${ }^{1}$ y Rosa E. Adrianzén ${ }^{1,2 *}$ \\ ${ }^{1}$ Instituto Regional de Oftalmología Javier Servat Univazo; ${ }^{2}$ Universidad Nacional de Trujillo. Trujillo, Perú
}

\begin{abstract}
Resumen
Objetivo: Determinar el efecto de la cirugía de catarata en la sensibilidad al contraste y calidad de vida de pacientes con diferentes tipos de catarata. Métodos: Estudio prospectivo, con diseño pretest-postest en pacientes con indicación de cirugía de catarata por facoemulsificación. Se evaluó la agudeza visual mejor corregida (AVMC) a diferentes niveles de contraste y la calidad de vida relacionada con la visión (CVRV) antes y después de 30 días de la cirugía. La población se dividió en tres grupos comparativos según el tipo de catarata predominante siguiendo la clasificación LOCS III (Lens Opacity Cataract System): nuclear, cortical y subcapsular posterior (SCP). Resultados: Se evaluaron 78 ojos de 78 pacientes con edad promedio $69.3 \pm 9.6$ (51-84) años. En el preoperatorio, la sensibilidad al contraste bajo estuvo alterada levemente en el grupo nuclear y severamente en el grupo SCP. El puntaje de la escala limitaciones de la CVRV fue significativamente menor para el grupo SCP comparado con el grupo nuclear (0 [0-15.6] vs. 6.2 [0-12.5]; $p=0.040$ ). Luego de la cirugía, la AVMC y la sensibilidad al contraste mejoraron en los tres grupos de estudio. Los cambios de mayor significancia se observaron en el grupo SCP. La mejoría en la CVRV se relacionó positivamente con la ganancia de agudeza visual en alto y bajo contraste en los tres grupos de estudio. Conclusión: La cirugía de catarata mejoró significativamente la sensibilidad al contraste y la calidad vida de todos los pacientes, especialmente en quienes presentaron opacidad SCP.
\end{abstract}

Palabras claves: Catarata. Agudeza visual. Calidad de vida. Sensibilidad de contraste. Facoemulsificación. Encuestas y cuestionarios.

\section{Abstract}

Objective: To determine the effect of cataract surgery on contrast sensitivity and quality of life of patients with different types of cataract. Methods: Prospective study with a pre- and post-test design in patients with indication for cataract surgery by phacoemulsification. Best-corrected visual acuity (BCVA) at different contrast levels and vision-related quality of life (V-RQOL) were evaluated before and 30 days after surgery. The study population was divided into 3 comparative groups according to the type of predominant cataract following the Lens Opacities Classification System III: nuclear, cortical and posterior subcapsular (PSC). Results: Seventy-eight eyes of 78 patients with a mean age of $69.3 \pm 9.6$ (51-86) years were included. 
In the preoperative period, low contrast sensitivity was slightly affected in the nuclear group and severely affected in the PSC group. V-RQOL limitations scale was significantly lower for the PSC group compared to the nuclear group (0 [0-15.6] vs. 6.2 [0-12.5]; $p=0.040$ ). After surgery, BCVA and contrast sensitivity improved in the three study groups. The most significant changes were observed in the PSC group. V-RQOL improvement was positively related to visual acuity gain in high and low contrast in the three study groups. Conclusion: Cataract surgery significantly improved contrast sensitivity and quality of life of all patients, especially in those with PSC opacity.

Key words: Cataract. Visual acuity. Quality of life. Contrast sensitivity. Phacoemulsification. Surveys and questionnaires.

\section{Introducción}

La discapacidad visual afecta a 285 millones de personas en el mundo, de las cuales, 39 millones están ciegas y 246 millones presentan baja visión ${ }^{1}$. De acuerdo con estudios realizados por la Organización Mundial de la Salud, la catarata es la causa más frecuente de ceguera recuperable en el mundo. Afecta principalmente a países en desarrollo y es responsable del $51 \%$ de la ceguera global, con cerca de 20 millones de personas ciegas ${ }^{2}$.

El deterioro de la visión ocasionado por el desarrollo de catarata se presenta de manera insidiosa, alterando progresivamente la agudeza visual (AV) de alto contraste, medida estándar de la función visual ${ }^{3}$. No obstante, el paciente puede presentar una marcada reducción de la sensibilidad al contraste a pesar de tener solo una pérdida de leve a moderada de la AV en alto contraste, y manifestar una reducida capacidad discriminatoria para identificar objetos o discriminar colores en ambientes poco iluminados, o presentar deslumbramiento en condiciones de iluminación indirecta (p. ej., días soleados o cuando se aproxima un automóvil con las luces encendidas) ${ }^{4,5}$. Estas alteraciones se presentarán en menor 0 mayor grado de acuerdo a la severidad y patrón específico de opacificación lenticular, y modificarán de forma variable la calidad de vida de los pacientes.

En la mayoría de hospitales públicos no se realizan de manera rutinaria pruebas de sensibilidad al contraste y calidad de vida en los pacientes con catarata a pesar de ser fáciles de aplicar y de bajo costo. Su implementación en la atención oftalmológica complementaría la evaluación de la función visual del paciente y permitiría identificar de forma más objetiva a los beneficiarios de un abordaje quirúrgico oportuno.

\section{Objetivo}

Determinar el efecto de la cirugía de catarata en la sensibilidad de contraste y calidad de vida de pacientes con diferentes tipos de catarata senil.

\section{Métodos}

\section{Diseño}

Se realizó un estudio prospectivo, de diseño pretest/postest, en pacientes con tres tipos diferentes de catarata, en quienes se evaluó la agudeza visual mejor corregida (AVMC) a varios niveles de contraste y su calidad de vida antes y después de la cirugía de catarata.

\section{Población y muestra}

El estudio fue realizado entre el 2 de enero y el 31 de julio de 2017 en el Instituto Regional de Oftalmología Javier Servat Univazo de la ciudad de Trujillo, Perú. El tamaño de la muestra se calculó mediante el análisis de varianza (ANOVA), considerando tres grupos de estudio, un riesgo alfa de 0.05 , un riesgo beta de 0.10 , una diferencia mínima a detectar de 15 puntos en la ganancia del puntaje total del NEI-VFQ-25 (National Eye InstituteVisual Function Questionnaire-25) ${ }^{6}$, desviación estándar de 5 puntos y un $50 \%$ de proporción prevista de pérdidas en el seguimiento. Se obtuvo una muestra de, como mínimo, 7 pacientes en cada grupo. Los pacientes fueron seleccionados a través de un muestreo consecutivo durante el periodo de estudio. Se incluyeron pacientes mayores a 50 años, de ambos sexos, con diagnóstico de catarata bilateral e indicación quirúrgica de facoemulsificación más implante de lente intraocular, con AVMC preoperatoria igual o peor a 20/200 y longitud axial entre 22.5 y $25 \mathrm{~mm}$. Se excluyeron del estudio pacientes con antecedentes de cirugía intraocular y con comorbilidades oculares y sistémicas de repercusión en su capacidad visual, como retinopatía diabética, degeneración macular, glaucoma, etc., y aquellos que presentaron complicaciones intraoperatorias y/o postoperatorias. Todos los pacientes firmaron un consentimiento informado asintiendo su participación en el estudio.

La población de estudio fue dividida en tres grupos (nuclear, cortical y subcapsular posterior [SCP]), según el tipo de catarata predominante del ojo con menor AVMC. La graduación de la severidad lenticular se basó en la clasificación LOCS III (Lens Opacity Cataract 
System) $)^{7}$. Debido a la dificultad de encontrar casos con opacificación exclusivamente cortical y SCP por su baja frecuencia, se permitió la coexistencia de opacificación nuclear < 2 para la conformación de estos grupos.

Antes y después de 30 días de la cirugía de catarata del primer ojo se evaluó en cada paciente la AVMC a $100 \%, 25 \%, 10 \%, 5 \%, 2.5 \%$ y $1.25 \%$ de nivel de contraste y la función visual y calidad de vida relacionada a la visión (CVRV). Estas evaluaciones fueron realizadas por uno de los investigadores (V.B.D), quien no participó de las cirugías.

Las cirugías fueron realizadas por tres cirujanos expertos en cirugía de catarata (P.C.S., J.H.P. y S.C.A.) y en todas ellas se implantó un lente intraocular monofocal, plegable, de una pieza y de diseño asférico (Tecnis ZCB00, Abbott Medical Optics, Inc., Santa Ana, CA).

\section{Agudeza visual en alto contraste}

La AVMC al $100 \%$ de contraste fue evaluada a 4 metros con la cartilla ETDRS logMAR (Precision Vision, Inc., La Salle, IL), en un cuarto de examen débilmente iluminado (161 lux). La altura de la cartilla fue estandarizada con la distancia de $124.46 \mathrm{~cm}$ entre la tercera fila de letras y el piso. La calificación de la AVMC con base en el número de letras leídas fue convertida a la unidad logMAR según el instructivo brindado por la Red de Investigación Clínica de Retinopatía Diabética (Diabetic Retinopathy Clinical Research Network) (disponible en: http://drcrnet.jaeb.org).

\section{Agudeza visual a bajo contraste y curvas de sensibilidad al contraste}

La AVMC para los contrastes 1.25\%, 2.5\%, 5\%, 10\% y $25 \%$ fue evaluada a 3 metros con cartillas de números de LEA de bajo contraste (Good-Lite Company, Elgin, IL), bajo las mismas condiciones de iluminación descritas para la AVMC en alto contraste. Con los datos encontrados se elaboraron curvas de sensibilidad al contraste pre y postoperatorias para cada tipo de catarata según lo propuesto por la casa fabricante (manual disponible en: https://www.good-lite.com).

\section{Función visual y la calidad de vida relacionada con la visión}

Se aplicó la versión en español del cuestionario NEI-VFQ-25ㄹ, el cual está compuesto por 12 escalas: salud general, visión general, salud mental, dolor ocular, visión cercana, visión lejana, visión periférica, desempeño social, visión de colores, conducción,
Tabla 1. Características demográficas y clínicas basales de pacientes con diferentes tipos de catarata

\begin{tabular}{|l|c|c|c|}
\hline $\begin{array}{c}\text { Nuclear } \\
(\mathbf{n}=32)\end{array}$ & $\begin{array}{c}\text { Cortical } \\
(\mathbf{n}=10)\end{array}$ & $\begin{array}{c}\text { SCP } \\
(\mathbf{n}=35)\end{array}$ \\
\hline $\begin{array}{l}\text { Edad (años) } \\
\text { (media + DE [R]) }\end{array}$ & $\begin{array}{c}74.4 \pm 7.4 \\
(51-84)\end{array}$ & $\begin{array}{c}70.2 \pm 9.2 \\
(52-82)\end{array}$ & $\begin{array}{c}64.9 \pm 8.5^{*} \\
(51-82)\end{array}$ \\
\hline Sexo M/F & $19 / 13$ & $6 / 4$ & $17 / 18$ \\
\hline Lateralidad 0D/OI & $15 / 17$ & $4 / 6$ & $14 / 21$ \\
\hline $\begin{array}{l}\text { LOCS III } \\
\text { (media + DE [R]) }\end{array}$ & $\begin{array}{c}2.7 \pm 0.6 \\
(2-4)\end{array}$ & $\begin{array}{c}3.6 \pm 0.8^{\dagger} \\
(2.5-5)\end{array}$ & $\begin{array}{c}3.9 \pm 0.6^{*} \\
(2.5-5)\end{array}$ \\
\hline
\end{tabular}

*Comparación SCP-nuclear, $\mathrm{p}<0.05$.

${ }^{\dagger}$ Comparación cortical-nuclear, $\mathrm{p}<0.05$.

DE: desviación estándar; F: femenino; LOCS: Lens Opacity Cataract System

M: masculino; OD: ojo derecho; OI: ojo izquierdo; R: rango; SCP: subcapsular posterior. ANOVA de 1 factor con corrección de Bonferroni.

limitaciones y dependencia. Este instrumento ha sido validado previamente para su uso en Perú en pacientes sometidos a cirugía de catarata ${ }^{9}$.

\section{Aspectos éticos}

El estudio se rigió por los principios éticos de la Declaración de Helsinki y fue aprobado por el Comité de Ética y de Investigación Institucional.

\section{Análisis estadístico}

El análisis estadístico fue realizado con el paquete SPSS versión 23 (IBM Corporation Inc., Armonk, NY). Se empleó estadística paramétrica y no paramétrica de acuerdo al cumplimiento o no del criterio de normalidad de los datos. Para el análisis intergrupos se utilizó el análisis de varianza (ANOVA de un factor) y el test de Kruskal Wallis con ajuste Dunn-Bonferroni. El cambio postoperatorio de las variables se evaluó a través de la prueba de los rangos con signo de Wilcoxon. Se realizó un análisis de correlación entre la variación postoperatoria del puntaje total del NEI-VFQ 25 y la variación de la AVMC a diferentes niveles de contraste, calculándose el coeficiente de correlación Rho $(\rho)$ de Spearman. Las diferencias fueron consideradas estadísticamente significativas cuando el valor $p$ fue $<0.05$.

\section{Resultados}

Cumplieron los criterios de inclusión y de exclusión 82 pacientes con catarata. Se perdieron 3 pacientes durante el seguimiento y 2 fueron eliminados por persistencia de 
Tabla 2. Agudeza visual preoperatoria (número de letras) a diferentes niveles de contraste según el tipo de catarata

\begin{tabular}{|c|c|c|c|c|c|c|c|}
\hline \multicolumn{8}{|c|}{ Contraste } \\
\hline & $\begin{array}{c}\text { Nuclear } \\
\text { \# optotipos } \\
\text { Mediana (RIO) }\end{array}$ & $\begin{array}{l}\text { Equiv } \\
\text { logMAR }\end{array}$ & $\begin{array}{c}\text { Cortical } \\
\text { \# optotipos } \\
\text { Mediana (RIO) }\end{array}$ & $\begin{array}{l}\text { Equiv } \\
\text { logMAR }\end{array}$ & $\begin{array}{c}\text { Posterior } \\
\text { \# optotipos } \\
\text { Mediana (RIO) }\end{array}$ & $\begin{array}{l}\text { Equiv } \\
\text { logMAR }\end{array}$ & $\mathbf{p}^{*}$ \\
\hline $1.25 \%$ & $0(0-9)^{\dagger}$ & - & 0 & - & 0 & - & 0.495 \\
\hline $2.5 \%$ & $0(0-17)^{\dagger}$ & - & $0(0-10)^{\dagger}$ & - & $0(0-10)^{\dagger}$ & - & 0.611 \\
\hline $5 \%$ & $1.5(0-9)$ & - & $0(0-5.3)$ & - & $0(0-3)$ & - & 0.204 \\
\hline $10 \%$ & $14(9.5-20.3)$ & 0.8 & $9(4.8-14)$ & 0.9 & $6(0-12)$ & 1 & $0.001^{\ddagger}$ \\
\hline $25 \%$ & $24(18.3-29)$ & 0.6 & $16.5(7.5-24.8)$ & 0.8 & $10(5-22)$ & 0.9 & $0.000^{\ddagger}$ \\
\hline $100 \%$ & $34(24-71.5)$ & 1.02 & $32(20-72)$ & 1.06 & $22(11-32)$ & 1.26 & $0.003^{\ddagger}$ \\
\hline
\end{tabular}

*Kruskal-Wallis con corrección Dunn-Bonferroni, $\mathrm{p}<0.05$ estadísticamente significativo.

${ }^{\dagger}$ Rango.

${ }^{\ddagger}$ Comparación subcapsular posteriores-nucleares.

RIQ: rango intercuartílico.

edema corneal a los 30 días postoperatorios, por lo que la muestra final fue constituida por 77 pacientes (grupo nuclear $=32$, grupo cortical $=10$, grupo posterior $=35$ ). Las características demográficas y clínicas en cada grupo de estudio se muestran en la Tabla 1. La edad promedio de los pacientes fue de $69.3 \pm 9.6(51-84)$ años. El grupo SCP fue más joven y presentó mayor grado de opacidad que los otros dos grupos.

En el preoperatorio, los pacientes del grupo nuclear leyeron más optotipos que aquellos con catarata cortical y SCP en todos los niveles de contraste. La AVMC al $100 \%, 25 \%$ y $10 \%$ de contraste fue significativamente menor en el grupo SCP comparada con la del grupo nuclear. Los pacientes con catarata nuclear discriminaron más optotipos a bajo contraste que aquellos con catarata cortical y SCP (Tabla 2, Fig. 1 A, B y C).

Luego de la cirugía de catarata, la AVMC mejoró para todos los niveles de contraste en los tres grupos de estudio. Los cambios en la AVMC de mayor significancia clínica y estadística se observaron en el grupo SCP (Tabla 3), representado por un mayor desplazamiento de la curva de sensibilidad a la derecha en comparación con los demás grupos (Fig. 1 C).

El puntaje total del VFQ-25 en el preoperatorio fue de 44.3 (35.9-50.7), 46.1 (35.2-56.5) y 45 (38.5-51.7) para los grupos nuclear, cortical y SCP, respectivamente $(p=0.670)$. En cuanto a las escalas del CVRV, el puntaje de la escala limitaciones fue significativamente menor en el grupo SCP comparado con el grupo nuclear (0 [0-15.6] vs. 6.2 [0-12.5]; $p=0.040$ ). Los puntajes en las demás escalas no mostraron diferencias entre los grupos. Luego de la cirugía, el puntaje total del VFQ-25 se incrementó en 24.5 (14.3-31.5), 25.8 (14.4-30.3) y $24.5(14.2-31.7)(p=0.992)$ puntos en los grupos nuclear, cortical y SCP, respectivamente. El puntaje de las escalas de CVRV se incrementó significativamente en los tres grupos de estudio, con la excepción de las escalas conducción y dolor ocular. El incremento en el puntaje de la escala visión lejana fue significativamente mayor en el grupo SCP comparado con el grupo nuclear $(p=0.023)$ (Tabla 4). El incremento del puntaje total del VFQ-25 en toda la población de estudio se correlacionó significativamente y de forma positiva con el incremento de la AVMC en los niveles de contraste $2.5 \%, 10 \%, 25 \%$ y $100 \%$ (Tabla 5).

\section{Discusión}

El desarrollo de catarata no solo produce cambios en la $\mathrm{AV}$, sino también alteraciones de otros componentes de la función visual como la sensibilidad al contraste y la estereopsis. Estas alteraciones suelen diferir según el tipo de catarata, y en determinados casos pueden manifestarse inclusive desde estadios tempranos de opacificación ${ }^{10,11}$. En este estudio evaluamos pacientes con cataratas que restringían la visión a $<20 / 200$ $(\log M A R=1)$. La opacificación de los grupos cortical y SCP fue significativamente superior a la del grupo nuclear, según este criterio de selección. La AVMC estuvo marcadamente reducida en el grupo de catarata SCP para todos los niveles de contraste, hecho que explica la mayor ganancia de AVMC luego de la cirugía. Al respecto, se sabe que las cataratas subcapsulares posteriores ocasionan una reducción de la sensibilidad 
Tabla 3. Agudeza visual a diferentes niveles de contraste pre y postoperatoria según el tipo de catarata

\begin{tabular}{|c|c|c|c|c|c|c|}
\hline \multicolumn{7}{|c|}{ Contraste } \\
\hline \multirow{2}{*}{$\begin{array}{l}\text { Tipo de } \\
\text { catarata }\end{array}$} & \multicolumn{2}{|c|}{ Preoperatoria } & \multicolumn{4}{|c|}{ Postoperatoria } \\
\hline & $\begin{array}{c}\text { N. }{ }^{0} \text { de } \\
\text { pacientes }\end{array}$ & $\begin{array}{l}\text { AVMC \# letras Mediana } \\
\text { (RIO) }\end{array}$ & $\begin{array}{c}\text { N. }{ }^{0} \text { de } \\
\text { pacientes }\end{array}$ & $\begin{array}{l}\text { AVMC \# Ietras Mediana } \\
\text { (RIO) }\end{array}$ & $\begin{array}{l}\text { Ganancia } \\
\text { AVMC }(\%)\end{array}$ & $\mathbf{p}^{*}$ \\
\hline \multicolumn{7}{|c|}{ Nucleares ( $n=32$ ) } \\
\hline $1.25 \%$ & 1 & $0(0-9)^{\dagger}$ & 13 & $0(0-10)$ & 13 & $0.001^{\ddagger}$ \\
\hline $2.5 \%$ & 4 & $0(0-17)^{\dagger}$ & 19 & $13.5(0-20)$ & 19 & $0.000^{\ddagger}$ \\
\hline $5 \%$ & 16 & $1.5(0-9)$ & 28 & $26(11.8-33.8)$ & 24.5 & $0.000^{\ddagger}$ \\
\hline $10 \%$ & 30 & $14(9.5-20.3)$ & 30 & $34(20.8-38.8)$ & $20(142 \%)$ & $0.000^{\ddagger}$ \\
\hline $25 \%$ & 32 & 24 (18.3-29) & 32 & $44(29.3-49.8)$ & $20(83 \%)$ & $0.000^{\ddagger}$ \\
\hline $100 \%$ & 32 & $34(24-71.5)$ & 32 & $82(73.3-82)$ & $48(141 \%)$ & $0.000^{\ddagger}$ \\
\hline \multicolumn{7}{|c|}{ Corticales $(n=10)$} \\
\hline $1.25 \%$ & 0 & 0 & 1 & $0(0-19)^{\dagger}$ & 0 & 0.317 \\
\hline $2.5 \%$ & 1 & $0(0-10)^{\dagger}$ & 7 & $5.5(0-22.5)$ & 5.5 & $0.018^{\ddagger}$ \\
\hline $5 \%$ & 3 & $0(0-5.3)$ & 10 & $23(9-35.3)$ & 23 & $0.008^{\ddagger}$ \\
\hline $10 \%$ & 9 & $9(4.8-14)$ & 10 & $36(19.5-46$ & $27(300 \%)$ & $0.005^{\ddagger}$ \\
\hline $25 \%$ & 9 & $16.5(7.5-24.8)$ & 10 & $44(30.8-53.8)$ & $27.5(167 \%)$ & $0.005^{\ddagger}$ \\
\hline $100 \%$ & 10 & $32(20-72)$ & 10 & $79.5(74.5-82)$ & $47.5(148 \%)$ & $0.005^{\ddagger}$ \\
\hline \multicolumn{7}{|c|}{ Subcapsulares posteriores ( $n=35$ ) } \\
\hline $1.25 \%$ & 0 & 0 & 13 & $0(0-8)$ & 0 & $0.001^{\ddagger}$ \\
\hline $2.5 \%$ & 2 & $0(0-10)^{\dagger}$ & 22 & $14(0-22)$ & 14 & $0.000^{\ddagger}$ \\
\hline $5 \%$ & 11 & $0(0-3)$ & 27 & $25(4-32)$ & 25 & $0.000^{\ddagger}$ \\
\hline $10 \%$ & 24 & $6(0-12)$ & 35 & $28(16-38)$ & $22(366 \%)$ & $0.000^{\ddagger}$ \\
\hline $25 \%$ & 33 & $10(5-22)$ & 35 & $44(23-52)$ & $34(340 \%)$ & $0.000^{\ddagger}$ \\
\hline $100 \%$ & 35 & $22(11-32)$ & 35 & $77(72-82)$ & $55(250 \%)$ & $0.000^{\ddagger}$ \\
\hline
\end{tabular}

al contraste para frecuencias espaciales bajas, inclusive antes que se produzca una esclerosis considerable del núcleo cristaliniano o una reducción franca de la AV de alto contraste ${ }^{12}$. Por este motivo se ha propuesto que la sensibilidad al contraste es un mejor indicador de discapacidad visual en cataratas en estadio temprano que la $A V^{10}$, sin embargo, aún es infrecuente su aplicación rutinaria en la práctica oftalmológica. Situación distinta es la que se observa con las cataratas nucleares, en las que la capacidad de discriminar a bajo contraste disminuye progresivamente a medida que se incrementa la opacificación, y guarda estrecha relación con la reducción de la $\mathrm{AV}$ en alto contraste ${ }^{11}$.

La mejoría en la calidad de vida de los pacientes se relacionó en este estudio con el incremento de la AV evaluada a diferentes niveles de contraste. El estudio de la sensibilidad al contraste es útil para definir el impacto de la catarata en la funcionabilidad del individuo y para estimar el riesgo ocupacional relacionado a una deficiente discriminación de los objetos en condiciones de baja iluminación. Un ejemplo de lo antes mencionado es lo reportado por Owsley, et al. ${ }^{13}$, 


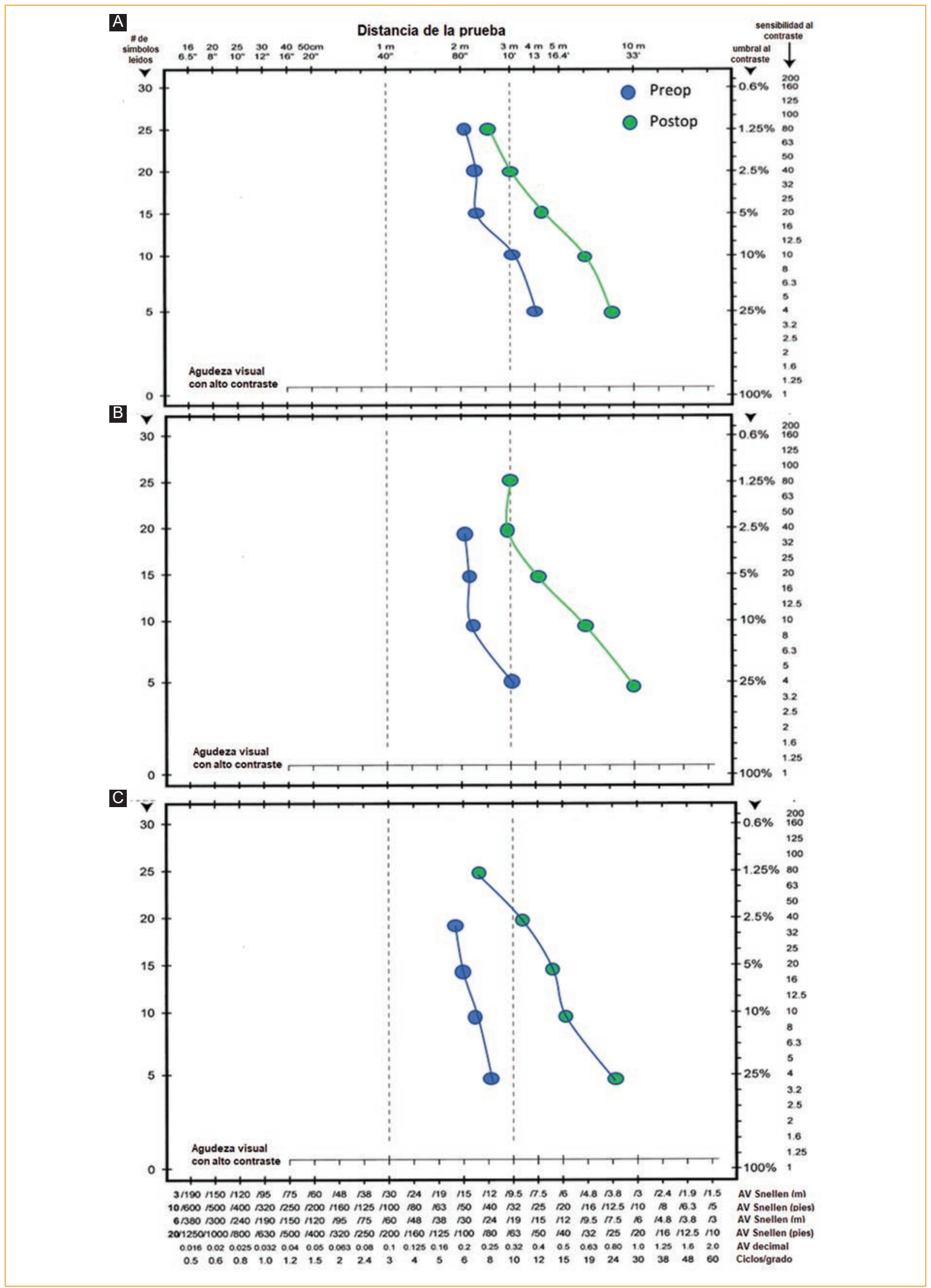

Figura 1. Curvas de sensibilidad de contraste pre y postoperatorias en el grupo nuclear (A), cortical (B) y subcapsular posterior (C). 
Tabla 4. Variación postoperatoria del puntaje de las escalas de calidad de vida relacionada con la visión evaluada con el VFO-25 según tipo de catarata

\begin{tabular}{|c|c|c|c|c|}
\hline \multicolumn{5}{|c|}{ Variación post-preoperatorio } \\
\hline VF0-25 & Nuclear Mediana (RIO) & Cortical Mediana (RIO) & SCP Mediana (RIO) & $\mathbf{p}^{*}$ \\
\hline SG & $25(25-25)$ & $25(0-25)$ & $25(0-25)$ & 0.789 \\
\hline VG & $20(20-40)$ & $20(20-20)$ & $20(20-40)$ & 0.519 \\
\hline SM & $31.3(18-37)$ & $28.1(12.5-39.1)$ & $25(12.5-37.5)$ & 0.923 \\
\hline DO & $0(-12.5-21.9)$ & $6.3(-15.6-12.5)$ & $-12.5(0-12.5)$ & 0.979 \\
\hline VC & $33(18.8-41.7)$ & $33.3(25-43.8)$ & $33.3(25-41.7)$ & 0.529 \\
\hline VL & $33(10.4-41.7)$ & $33.3(6.3-41.7)$ & $33.3(8.3-41.7)$ & $0.023^{\dagger}$ \\
\hline VP & $25(0-25)$ & $25(0-25)$ & $25(0-50)$ & 0.959 \\
\hline DS & $25(0-50)$ & $25(-12.5-37.5)$ & $0(-25-50)$ & 0.762 \\
\hline VCo & $25(0-43.8)$ & $25(18.8-50)$ & $25(0-50)$ & 0.732 \\
\hline C & $0(0-3.1)$ & $0(0-1)$ & $0(0-8.3)$ & 0.980 \\
\hline L & $37.5(37.5-50)$ & $50(37.5-65.6)$ & $37.5(37.5-62.5)$ & 0.383 \\
\hline D & $16.7(0-33.3)$ & $20(16.7-33.3)$ & $25(8.3-33.3)$ & 0.648 \\
\hline Total & $24.6(14.3-31.5)$ & $25.8(14.4-30.3)$ & $24.5(14.2-31.7)$ & 0.992 \\
\hline
\end{tabular}

Tabla 5. Correlación entre la variación del puntaje total del NEI-VFO-25 y la variación postoperatoria de la AVMC en alto y bajo contraste

\begin{tabular}{|l|c|c|}
\hline$\triangle$ Puntaje total NEI-VF0-25 & Coeficiente Rho $(p)$ & Valor $p$ \\
\hline$\triangle$ AVMC 1.25\% & 0.2 & 0.109 \\
\hline AVMC 2.5\% & 0.3 & 0.009 \\
$\triangle$ AVMC 5\% & 0.2 & 0.114 \\
$\triangle$ AVMC 10\% & 0.3 & 0.006 \\
$\triangle$ AVMC 25\% & 0.3 & 0.007 \\
\hline AVMC 100\% & 0.5 & 0.000 \\
\hline
\end{tabular}

Correlación de Spearman, $\mathrm{p}<0.05$, estadísticamente significativo.

AVMC: agudeza visual mejor corregida. NEI-VFO-25: National Eye Institute-Visual Function Questionnaire-25.

quienes indican que la sensibilidad al contraste es un factor independiente de la AV para la generación de accidentes de tránsito en pacientes con catarata, remarcando la importancia de su evaluación en la toma de decisiones terapéuticas. La sensibilidad al contraste, por tanto, debe considerarse en práctica oftalmológica habitual como un indicador del deterioro de la función visual más sensible que la evaluación convencional de la $A V$ en pacientes con catarata.
En cuanto a las escalas de evaluación de la CVRV, observamos que los pacientes con catarata SCP presentaban mayores limitaciones en la realización de actividades de la vida diaria, probablemente debido, en parte, al deterioro significativo de la sensibilidad al contraste. Otros estudios han reportado que la CVRV en pacientes con catarata se relaciona más estrechamente con los cambios en la sensibilidad de contraste y la estereopsis que con el cambio en la $\mathrm{AV}^{1,6,14,15}$.

Luego de la cirugía, observamos un incremento significativo en la mayoría de las escalas del VFQ-25 en cada uno de los subtipos de catarata. Esta mejoría en la función visual fue estadísticamente superior para el grupo SCP cuando se lo comparó con el nuclear para la escala visión lejana. Esta diferencia no encontrada en el preoperatorio podría explicarse no solo por la ganancia de AVMC en alto contraste sino también por la importante mejoría en la sensibilidad al contraste observada en el grupo SCP. No obstante, es necesario resaltar que el cambio en la calidad de vida depende de muchos factores, entre ellos la edad, la salud física y emocional, el estado cognitivo, la influencia de otras comorbilidades y el entorno social del paciente. En este sentido, el VFQ-25 no solo evalúa las limitaciones para realizar tareas que dependen de 
la salud visual, sino también la salud mental y desempeño social del individuo, lo cual lo convierte en un mejor indicador de satisfacción del desempeño visual que la $\mathrm{AV}^{14}$. Resultados semejantes a los nuestros fueron reportados por To, et al. ${ }^{6}$ e Ishii, et al. ${ }^{16}$, quienes además señalaron que la mejoría en la CVRV posquirúrgica se relaciona estrechamente con la mejoría del estado cognitivo y depresivo de los pacientes. Por otro lado, es preciso indicar que al ser el VFQ-25 un cuestionario no específico para el tipo de padecimiento, la escala dolor no mostró una variación significativa, ya que la cirugía de catarata no supone una agresión grave para el ojo, y es de recuperación rápida con mínimas molestias ${ }^{9,17}$. Asimismo, el puntaje para la escala conducción no sufrió un cambio relevante luego de la cirugía, ya que en la población de estudio la conducción de automóviles no es habitual, por limitantes como la edad, estado socioeconómico y nivel educativo de los pacientes atendidos en nuestro establecimiento de salud.

Como limitaciones en nuestro estudio podemos mencionar el posible sesgo de medición generado por el evaluador de la AV y la CVRV, ya que fue investigador y, por lo tanto, no se encontraba ciego para el estudio. Por otro lado, debido a la baja frecuencia de cataratas a predominio cortical, el muestreo durante el periodo de estudio permitió seleccionar únicamente 10 pacientes para este grupo. A futuro, podría considerarse la inclusión de pacientes con opacidad nucleocortical y corticoposterior para evaluar indirectamente el efecto de la opacidad cortical en los parámetros de evaluación. Nuestros resultados solo alcanzan a reportar los beneficios de la cirugía monocular de catarata sobre la sensibilidad al contraste y la CVRV. Un complemento a estos resultados sería la evaluación de los efectos de la cirugía bilateral de catarata, la cual ha demostrado en otros estudios beneficios adicionales en la función visual de los pacientes ${ }^{6,18}$.

El abordaje del paciente con catarata debe realizarse de manera integral, considerando tanto aspectos clínicos como la función visual en actividades del mundo real. Una marcada limitación de la capacidad de realizar actividades de la vida diaria a consecuencia de la visión sería el sustento para un abordaje quirúrgico temprano. Por otro lado, las dificultades en la aplicación de los cuestionarios de función visual deben ser consideradas para implementar cuestionarios específicos a la patología evaluada, tomando en cuenta las variantes socioculturales de las poblaciones.

Debido a sus implicancias en la toma de decisiones, recomendamos incluir la evaluación de la sensibilidad de contraste y la calidad de vida relacionada de forma rutinaria en el paciente con catarata, ya que brindan mayor información sobre la discapacidad de los pacientes en sus actividades de la vida diaria.

\section{Conclusiones}

En este estudio, la cirugía de catarata mejoró significativamente la AV de alto y bajo contraste y la calidad de vida de todos los pacientes evaluados.

Los pacientes con catarata subcapsular posterior presentaron menor sensibilidad al contraste y mayor limitación para realizar actividades a consecuencia de su visión que los pacientes con catarata nuclear.

La mejoría postoperatoria de la AV y la sensibilidad al contraste fue sustancialmente superior en pacientes con catarata SCP, así como su percepción de mejoría en la realización de actividades utilizando su visión a distancia.

Los cuestionarios de calidad de vida como el VFQ-25 deben adaptarse culturalmente para poder aplicarlos objetivamente en nuestra población.

\section{Conflicto de intereses}

Los autores declaran no tener conflictos de interés con la formulación empleada en este estudio.

\section{Financiamiento}

Los autores no han recibido patrocinio para llevar a cabo este estudio.

\section{Responsabilidades éticas}

Protección de personas y animales. Los autores declaran que para esta investigación no se han realizado experimentos en seres humanos ni en animales.

Confidencialidad de los datos. Los autores declaran que en este artículo no aparecen datos de pacientes.

Derecho a la privacidad y consentimiento informado. Los autores declaran que en este artículo no aparecen datos de pacientes.

\section{Bibliografía}

1. Acosta R, Hoffmeister L, Román R, Comas M, Castilla M, Castells $X$. Revisión sistemática de estudios poblacionales de prevalencia de catarata. Arch Soc Esp Oftalmol. 2006;81(9):509-16.

2. WHO I Priority eye diseases [Internet]. WHO. Disponible en: http://www. who.int/blindness/causes/priority/en/

3. Kessel L, Andresen J, Erngaard D, Flesner P, Tendal B, Hjortdal J. Indication for cataract surgery. Do we have evidence of who will benefit 
from surgery? A systematic review and meta-analysis. Acta Ophthalmol (Copenh). 2016;94(1):10-20.

4. Sniatecki JJ, Styles C, Boyle N, Sanders R. Cataract surgery: factors influencing decision to treat and implications for training (south-east Scotland 2008-2014). Clin Ophthalmol Auckl NZ. 2015;9:1821-7.

5. Ginsburg AP. Contrast sensitivity: determining the visual quality and function of cataract, intraocular lenses and refractive surgery. Curr Opin Ophthalmol. 2006;17(1):19.

6. To KG, Meuleners LB, Fraser ML, Do DV, Duong DV, Huynh V-AN, et al The impact of cataract surgery on vision-related quality of life for bilatera cataract patients in Ho Chi Minh City, Vietnam: a prospective study. Health Qual Life Outcomes. 2014;12:16.

7. Chylack LT, Wolfe JK, Singer DM, Leske MC, Bullimore MA, Bailey IL, et al. The Lens Opacities Classification System III. Arch Ophthalmol. 1993:111(6):831-6.

8. Mangione CM, Lee PP, Gutierrez PR, Spritzer K, Berry S, Hays RD. Development of the 25-list-item National Eye Institute Visual Function Questionnaire. Arch Ophthalmol. 2001;119(7):1050-8.

9. Luján Paredes S, Pizango Malqui O, Alburquerque Duglio M, Valenzuela Tito $M$, Mayta-Tristán $P$. Variación de la función visua y calidad de vida luego de cirugía de catarata por facoemulsificación con implante de lente intraocular. Rev Mex Oftalmol. 2014;88(4): 176-81.

10. Elliott DB, Situ P. Visual acuity versus letter contrast sensitivity in early cataract. Vision Res. 1998;38(13):2047-52.
11. Stifter E, Sacu S, Thaler A, Weghaupt H. Contrast acuity in cataracts of different morphology and association to self-reported visual function. Invest Ophthalmol Vis Sci. 2006;47(12):5412-22.

12. Cheng CY, Yen MY, Chen SJ, Kao SC, Hsu WM, Liu JH. Visual acuity and contrast sensitivity in different types of posterior capsule opacification. J Cataract Refract Surg. 2001;27(7):1055-60.

13. Owsley C, Stalvey BT, Wells J, Sloane ME, McGwin G. Visual risk factors for crash involvement in older drivers with cataract. Arch Ophthalmol Chic III 1960. 2001;119(6):881-7.

14. Stifter $E$, Sacu $S$, Weghaupt $H$. Functional vision with cataracts of different morphologies: comparative study. J Cataract Refract Surg. 2004;30(9):1883-91.

15. Datta S, Foss AJE, Grainge MJ, Gregson RM, Zaman A, Masud T, et al. The importance of acuity, stereopsis, and contrast sensitivity for health-related quality of life in elderly women with cataracts. Invest Ophthalmol Vis Sci. 2008;49(1):1-6.

16. Ishii K, Kabata T, Oshika T. The impact of cataract surgery on cognitive impairment and depressive mental status in elderly patients. Am J Ophthalmol. 2008;146(3):404-9.

17. Cabezas-León M, Gracia-San Román J, García-Caballero J, Morente-Matas P. Calidad de vida en pacientes intervenidos de catarata. Arch Soc Esp Oftalmol. 2005;80(8):449-56.

18. Castells X, Comas M, Alonso J, Espallargues M, Martínez V, García-Arumí J, et al. In a randomized controlled trial, cataract surgery in both eyes increased benefits compared to surgery in one eye only. J Clin Epidemiol. 2006;59(2):201-7. 